\title{
Polydatin supplementation ameliorates diet-induced development of insulin resistance and hepatic steatosis in rats
}

\author{
QI ZHANG ${ }^{1 *}$, YINGYING TAN $^{1 *}$, NAN ZHANG $^{1}$ and FANRONG YAO ${ }^{2}$ \\ ${ }^{1}$ Department of Physiology, Basic Medical College, Shaanxi University of Chinese Medicine, Xianyang, \\ Shaanxi 712046, P.R. China; ${ }^{2}$ Department of Pharmacology and Toxicology, Brody School of Medicine, \\ East Carolina University, Greenville, NC 27834, USA
}

Received December 12, 2013; Accepted September 4, 2014

DOI: $10.3892 / \mathrm{mmr} .2014 .2708$

\begin{abstract}
The pathophysiology of non-alcoholic fatty liver disease remains to be elucidated, and the currently available treatments are not entirely effective. Polydatin, a stilbenoid compound derived from the rhizome of Polygonum cuspidatum, has previously been demonstrated to possess hepatoprotective effects. The present study aimed to determine the effects of polydatin supplementation on hepatic fat accumulation and injury in rats fed a high-fat diet. In addition, the mechanisms underlying the protective effects of polydatin were examined. Male Sprague Dawley rats were randomly divided into four groups and received one of four treatment regimes for 12 weeks: Control diet, control diet supplemented with polydatin, high-fat diet, or high-fat diet supplemented with polydatin. Polydatin was supplemented in the drinking water at a concentration of $0.3 \%$ (wt/vol). The results of the present study showed that long-term high-fat feeding resulted in fatty liver in rats, which was manifested by excessive hepatic neutral fat accumulation and elevated plasma alanine aminotransferase and aspartate aminotransferase levels. Polydatin supplementation alleviated the hepatic pathological changes, and attenuated the insulin resistance, as shown by an improved homeostasis model assessment of basal insulin resistance values and a glucose tolerance test. Polydatin supplementation also corrected abnormal leptin and adiponectin levels. Specifically, polydatin supplementation enhanced insulin sensitivity in the liver, as shown by improved insulin receptor substrate 2 expression levels and Akt phosphorylation in the rat liver, following
\end{abstract}

Correspondence to: Dr Qi Zhang, Department of Physiology, Basic Medical College, Shaanxi University of Chinese Medicine, 1 Century Avenue, Xianyang, Shaanxi 712046, P.R. China

E-mail: zhangndsu@yahoo.com

*Contributed equally

Key words: polydatin, fatty liver, insulin resistance, adipokine, insulin receptor substrate, leptin, hepatic steatosis high-fat diet feeding. The results of the present study suggest that polydatin protects rats against high-fat feeding-induced insulin resistance and hepatic steatosis. Polydatin may be an effective hepatoprotective agent and a potential candidate for the prevention of fatty liver disease and insulin resistance.

\section{Introduction}

Nonalcoholic fatty liver disease (NAFLD) is defined as the presence of increased fat in the liver that is not the result of excessive alcohol consumption. It is comprised of a morphological spectrum of liver injuries, ranging from simple triglyceride (TG) accumulation in the cytoplasm of hepatocytes (steatosis), to inflammatory and hepatocellular injury (nonalcoholic steatohepatitis), which may lead to fibrosis and cirrhosis (1-3). Due to its high prevalence in association with obesity, diabetes and insulin resistance, NAFLD is increasingly being considered as a hepatic manifestation of the metabolic syndrome (4). The exact cause of NAFLD remains unknown; however, both obesity and insulin resistance have been shown to have a role in the disease process $(5,6)$. Fat accumulation in the liver results from an imbalance between the uptake, synthesis, export and oxidation of fatty acids. In normal circumstances, insulin inhibits free fatty acid (FFA) release from adipose tissues. However, as insulin resistance develops, the elevated plasma concentrations of glucose and fatty acids promotes hepatic fatty acid synthesis and impairs $\beta$-oxidation, resulting in the formation of hepatic steatosis. Hepatic steatosis conversely exacerbates the degree of insulin resistance, and accelerates the subsequent transition to steatohepatitis and fibrosis $(7,8)$.

There is currently no effective or generally accepted treatment for NAFLD. Polydatin is a stilbenoid compound derived from the rhizome of Polygonum cuspidatum (9). This plant has previously been used in traditional Chinese medicine, to treat digestive disorders and ischemia/reperfusion injury $(9,10)$. One of the main properties of polydatin is its hepatoprotective activity, which has been reported to induce gallbladder contraction, prevent biliary cholesterol-stone formation and protect against tetrachloromethane and aflatoxin B1 hepatotoxicity $(9,11-13)$. In addition, it has been demonstrated that 
polydatin suppresses the oxidative and inflammatory damage in ischemic stroke (14). Furthermore, polydatin treatment has been shown to significantly reduce the serum levels of total cholesterol (TC) and TGs in hyperlipidemic hamsters and rabbits, induced by a high-fat diet $(15,16)$. A previous study demonstrated that polydatin alleviated hepatic fat accumulation, by reducing oxidative damage in the liver and regulating the expression of genes associated with hepatic fatty acid biosynthesis (17). Despite these hypolipidemic and antioxidant activities, the extent to which polydatin improves hepatic steatosis has yet to be elucidated.

In the present study, NAFLD with insulin resistance was induced in male rats by feeding them a nutritionally complete high-fat diet. The aim of the present study was to determine whether polydatin, when administered orally with a high-fat diet, would prevent hepatic lipid accumulation and reverse insulin resistance.

\section{Materials and methods}

Ethical approval. All animal experiments undertaken in the present study were performed in strict accordance with international ethical guidelines and the National Institutes of Health (NIH) Guide concerning the Care and Use of Laboratory Animals (NIH, Bethesda, MA, USA). The experiments were performed with the approval of the Committee of Experimental Animal Administration of the Shaanxi University of Chinese Medicine (permit number: 10076; Xianyang, China).

Animal model and experimental protocol. Male Sprague Dawley rats, weighing 160-180 g, were purchased from the Experimental Animal Center of Xi'an Jiaotong University (Xi'an, China). The rats were housed at $24^{\circ} \mathrm{C}$, with a $12 \mathrm{~h}$ light-dark cycle and free access to food and water. The animal experiments were approved by the Committee of Experimental Animal Administration of Shaanxi University of Chinese Medicine. Initially, the rats were housed in conventional conditions and fed a standard diet and water ad libitum at the animal facility for one week prior to the experiments. Thereafter, the rats were divided into four groups ( $n=8 /$ group) housed in individual cages, and administered one of four treatments: Control diet (Con), control diet supplemented with polydatin (CP), high-fat diet (HF), and high-fat diet supplemented with polydatin (HP). Both the control $(77.0 \%$ carbohydrate, $5.0 \%$ fat, $18.0 \%$ protein) and high-fat pellet diet protocols $(58.0 \%$ fat, $24.0 \%$ carbohydrate, $18.0 \%$ protein) were obtained from the Experimental Animal Center of Xi'an Jiaotong University (Xi'an, China). The lipids were in the form of saturated $(0.9$ and $30.4 \mathrm{~g} / 100 \mathrm{~g}$ diet $)$ and unsaturated (4.6 and $5.3 \mathrm{~g} / 100 \mathrm{~g} \mathrm{diet)} \mathrm{fat} \mathrm{in} \mathrm{the} \mathrm{control} \mathrm{and} \mathrm{high-fat} \mathrm{diet}$ groups, respectively. Polydatin (3,4',5-trihydroxystilbene-3- $\beta$ mono-D-glucoside; purity, $>99 \%$ ) was obtained from Suzhou Baozetang Biotechnology Co., Ltd. (Suzhou, China). Polydatin was supplemented in the drinking water at a concentration of $0.3 \%$ (wt/vol) and administered simultaneously with the high-fat diet feeding. In all of the animals, body weight and food intake were measured every other day. Food and water were supplied ad libitum. Food intake was determined by measuring the difference between the weight of the study diets provided, and the weight of the food at the end of a $24 \mathrm{~h}$ period. Spillage was checked throughout the experiment and was considered negligible. The rats were maintained on the treatment regimes for 12 weeks, prior to being sacrificed. At the end of the experiment, the rats were sacrificed by $\mathrm{CO}_{2}$ inhalation following a $12 \mathrm{~h}$ fasting period, and blood samples were collected from the femoral artery and stored as plasma at $-80^{\circ} \mathrm{C}$, until further use. The intact livers were harvested and weighed. The liver index was calculated as the liver/body weight ratio. The epididymal fat was removed in order to measure visceral adiposity. Sections from the right lobe of the liver were washed in cold saline and placed in $10 \%$ formalin solution for histopathological analysis. The other samples were immediately frozen in liquid nitrogen, and stored at $-80^{\circ} \mathrm{C}$ until further use.

Histological analysis. The liver sections were paraffin-embedded, sliced into $5 \mu \mathrm{m}$ sections and stained with hematoxylin \& eosin (H\&E), as described by previous methods (17). The pathological changes were assessed and photographed using an Olympus BX-51 microscope (Olympus Corporation, Tokyo, Japan). The liver biopsies were scored according to Brunt et al (18), as follows: 0, no steatosis; 1, fatty hepatocytes occupying $<10 \%$ of the parenchyma; 2 , between 10 and $30 \%$; 3, between 30 and 60\%; and 4, fatty hepatocytes occupying $>60 \%$ of the parenchyma. Liver pathology was assessed in a blinded manner by two independent pathologists with expertise in rodent liver histology.

Biochemical assays. The activities of alanine aminotransferase (ALT) and aspartate aminotransferase (AST) were measured in the plasma using commercially available enzyme assay kits (Wako Pure Chemical Industries Ltd., Osaka, Japan). Plasma TG, TC, and FFA concentrations were determined using enzymatic reagent kits from Biosino Bio-Technology and Science Inc. (Beijing, China), according to the manufacturer's instructions. Hepatic lipids were extracted using chloroform-methanol (2:1), as previously described by Folch et al (19), and then dissolved using Triton X-100. The hepatic concentrations of TG and TC were determined using the enzymatic reagent kits (Biosino Bio-Technology and Science Inc.), as previously described (17,20).

Glucose tolerance test. Glucose tolerance tests were conducted $1 \mathrm{wk}$ prior to the end of feeding. Following an $8 \mathrm{~h}$ fasting period, the rats were anesthetized, and, after the collection of an unchallenged sample (time 0), a solution of 50\% glucose $(2.0 \mathrm{~g} / \mathrm{kg}$ body wt) was administrated by oral gavage. During the test, blood was collected from the tail vein at 30,60 and 120 min following glucose administration. All of the blood glucose measurements were performed using a hand-held glucometer (Roche Diagnostics, Shanghai, China).

Plasma insulin, leptin, and adiponectin ELISA. Plasma insulin, leptin and adiponectin levels were measured using commercially available ELISA kits (Crystal Chem Inc., Downers Grove, IL, USA), according to the manufacturer's instructions.

The homeostasis model assessment of basal insulin resistance (HOMA-IR) was used to calculate an index 
from the product of the concentration of fasting plasma glucose $(\mathrm{mmol} / \mathrm{l})$ and plasma insulin $(\mu \mathrm{U} / \mathrm{ml})$, divided by 22.5 (21). Lower HOMA-IR values were considered to indicate a greater insulin sensitivity, whereas higher HOMA-IR values were considered to indicate a lower insulin sensitivity, or insulin resistance.

Quantitative polymerase chain reaction ( $q P C R$ ). A qPCR was performed to detect changes in the mRNA expression levels of insulin receptor (IR), insulin receptor substrate-1 (IRS-1) and -2 (IRS-2) in the liver tissues, as described by previous methods $(17,22)$. The extraction of total RNA, from the liver tissues, was performed using the RNeasy Mini kit (Qiagen, Valencia, CA, USA), according to the manufacturer's instructions. The purity and concentration of the RNA samples were determined spectrophotometrically (SpectraMax M5 microplate spectrophotometer; Molecular Devices, LLC., Sunnyvale, CA, USA). First, $2 \mu \mathrm{g}$ of total RNA was reverse transcribed into cDNA using Reverse Transcriptase (Qiagen). Contaminating genomic DNA was eliminated using DNase I (Qiagen). The qPCR reactions were conducted by placing $10 \mu \mathrm{l}$ cDNA into 96-well plates with the TaqMan PCR Master mix (Applied Biosystems Life Technologies, Foster City, CA, USA). The specific primers and probes used for detection of IR, IRS-1 and IRS-2 mRNA (Gene Expression Products: IR, Rn00567070_m1; IRS1, Rn04244524_m1; IRS2, Rn01482270_s1; and $\beta$-actin, Rn00667869_m1) were obtained from Applied Biosystems Life Technologies, and the qPCR was performed using an Applied Biosystems PRISM 7000 sequence detection system, according to the manufacturer's instructions (Applied Biosystems Life Technologies). A fluorescent comparative cycle threshold (Ct) method was used to quantify mRNA expression levels, with $\beta$-actin used as the internal control. The results of the qPCR were expressed as the ratio of the mRNA of interest to $\beta$-actin.

Western blotting. The liver tissues were homogenized in lysis buffer containing $20 \mathrm{mM}$ Tris $\cdot \mathrm{HCl}(\mathrm{pH} 6.8), 150 \mathrm{mM} \mathrm{NaCl}$, $10 \%$ glycerol, $1 \% \mathrm{NP}-40$, and $8 \mu \mathrm{l} / \mathrm{ml}$ inhibitor cocktail (125 mM phenylmethylsulfonyl fluoride, $2.5 \mathrm{mg} / \mathrm{ml}$ aprotinin, $2.5 \mathrm{mg} / \mathrm{ml}$ leupeptin, $2.5 \mathrm{mg} / \mathrm{ml}$ antipain, and $2.5 \mathrm{mg} / \mathrm{ml}$ chymostatin). The tissue lysates were centrifuged at $15,000 \mathrm{x} \mathrm{g}$ for 10 mins at $4^{\circ} \mathrm{C}$, and the supernatants were collected. The protein concentration was determined using a Protein Assay kit (Bio-Rad Laboratories, Hercules, CA, USA). The samples were boiled for $5 \mathrm{~min}$, and separated by $8 \%$ SDS-PAGE $(10 \mu \mathrm{g}$ of protein, $20 \mu \mathrm{l}$ per well) using Bio-Rad Minigel apparatus, at $100 \mathrm{~V}$ for $60 \mathrm{~min}$ (Bio-Rad Laboratories. The fractioned proteins on the gel were electrophoretically transferred to nitrocellulose membranes (Millipore, Billerica, MA, USA), at $350 \mathrm{~mA}$ for $90 \mathrm{~min}$. Following blocking with $5 \%$ skimmed milk, the membranes then were hybridized at $4^{\circ} \mathrm{C}$ overnight with the following primary antibodies: polyclonal rabbit anti-IRS2 (1:500), polyclonal rabbit anti-p-AKT (1:500), polyclonal rabbit anti-AKT (1:500) and polyclonal goat antiGAPDH (1:500), which were all purchased from Santa Cruz Biotechnology (Dallas, TX, USA). The membranes were washed with phosphate-buffered saline with Tween 20 (Sigma-Aldrich, St. Louis, MO, USA) and further incubated with the secondary antibody, horseradish peroxidase-conjugated immunoglobulin G (1:2,000; Santa Cruz Biotechnology), at $37^{\circ} \mathrm{C}$ for $60 \mathrm{~min}$. Immunoreactivity was detected using a chemiluminescence reagent kit (GE Healthcare, Little Chalfont, UK), for $5 \mathrm{~min}$ at room temperature. The exposed bands were analyzed using the Quantity One Software (Bio-Rad Laboratories).

Statistical analyses. All of the data are expressed as the means \pm standard error. Statistical analyses were performed using a one-way analysis of variance, and further analyzed using the Newman-Keuls test for statistical difference. A $\mathrm{P}<0.05$ was considered to indicate a statistically significant difference.

\section{Results}

Changes in body weight and food consumption. Following the 12 week feeding regimes, the absolute body weight of each rat from each group was measured (Table I). There were no significant differences in the absolute body weight of the rats between the Con and CP groups, at the end of the feeding period. The rats in the HF group had a significantly larger body weight and greater body weight gain, as compared with the Con group $(\mathrm{P}<0.05)$. The increase in body weight, in response to a high-fat diet, was associated with the development of visceral obesity, as shown by the progressive increase in epididymal fat weight. Supplementation of polydatin in the HF group (HP) had no significant effects on body weight gain, but reduced the weight of epididymal fat, as compared with the HF group $(\mathrm{P}<0.05)$. The consumption of food was monitored every other day, and no significant differences were observed in the daily food consumptions among the groups.

Polydatin supplementation alleviates high-fat diet-induced fatty liver and liver injury. The hepatic pathological alterations from the four groups were evaluated by measuring hepatic triglyceride contents and circulating liver enzyme levels. A long-term high-fat diet induced liver injury and fatty liver in the rats, which manifested as increased plasma ALT and AST levels and hepatic triglyceride contents (Table I). In response to polydatin supplementation in the drinking water, both hepatic TG accumulation and liver injury were significantly reduced in the high-fat diet fed group $(\mathrm{P}<0.05)$. Furthermore, histological sections of the liver from each experimental group were observed by $\mathrm{H} \& \mathrm{E}$ staining. Following a 12 week high-fat diet, the rats demonstrated severe hepatic microvesicular and macrovesicular fat, whereas polydatin supplementation significantly eliminated hepatic steatosis $(\mathrm{P}<0.05)$ (Fig. 1).

Polydatin supplementation alleviates insulin resistance. To determine whether the hepatoprotective effects of polydatin were associated with improved insulin resistance in the high-fat diet fed rat model, the insulin sensitivity of the rats was examined by measuring HOMA-IR, glucose tolerance, and plasma FFA levels. High-fat diet feeding resulted in a significantly higher HOMA-IR $(\mathrm{P}<0.01)$ (Fig. 2A), severe hyperglycemia upon glucose administration, and impaired glucose tolerance $(\mathrm{P}<0.05)($ Fig. $2 \mathrm{~B})$, as well as significantly increased circulating FFA levels $(\mathrm{P}<0.05)$ (Table I), 
Table I. Body weight, liver index and biochemical parameters of the experimental rats.

\begin{tabular}{|c|c|c|c|c|}
\hline & Con & $\mathrm{CP}$ & $\mathrm{HF}$ & $\mathrm{HP}$ \\
\hline Body Weight (g) & $482.37 \pm 16.53$ & $474.13 \pm 17.12$ & $537.08 \pm 17.45^{\mathrm{a}}$ & $491.52 \pm 16.27$ \\
\hline Liver index & $3.12 \pm 0.27$ & $2.97 \pm 0.24$ & $5.04 \pm 0.32^{\mathrm{b}}$ & $3.68 \pm 0.26^{\mathrm{d}}$ \\
\hline Epididymal fat & $1.43 \pm 0.12$ & $1.39 \pm 0.11$ & $2.25 \pm 0.13^{b}$ & $1.78 \pm 0.09^{c}$ \\
\hline \multicolumn{5}{|l|}{ Plasma } \\
\hline $\operatorname{ALT}(\mathrm{U} / \mathrm{l})$ & $52.11 \pm 3.13$ & $49.73 \pm 3.81$ & $82.37 \pm 5.49^{b}$ & $57.83 \pm 4.25^{\mathrm{d}}$ \\
\hline AST (U/l) & $63.51 \pm 4.31$ & $56.83 \pm 5.72$ & $134.38 \pm 8.02^{\mathrm{b}}$ & $76.32 \pm 3.66^{d}$ \\
\hline $\mathrm{TG}(\mathrm{mmol} / \mathrm{l})$ & $0.68 \pm 0.08$ & $0.73 \pm 0.09$ & $1.28 \pm 0.08^{b}$ & $0.98 \pm 0.06^{\mathrm{ac}}$ \\
\hline $\mathrm{TC}(\mathrm{mmol} / \mathrm{l})$ & $1.61 \pm 0.14$ & $1.69 \pm 0.21$ & $2.87 \pm 0.13^{b}$ & $2.19 \pm 0.09^{\mathrm{ac}}$ \\
\hline FFA (mmol/l) & $0.84 \pm 0.04$ & $0.91 \pm 0.06$ & $1.28 \pm 0.05^{\mathrm{b}}$ & $0.96 \pm 0.07^{\mathrm{d}}$ \\
\hline \multicolumn{5}{|l|}{ Liver } \\
\hline $\mathrm{TG}(\mu \mathrm{mol} / \mathrm{g})$ & $26.37 \pm 2.13$ & $25.02 \pm 2.21$ & $39.63 \pm 3.02^{\mathrm{b}}$ & $29.35 \pm 2.79^{d}$ \\
\hline $\mathrm{TC}(\mu \mathrm{mol} / \mathrm{g})$ & $6.08 \pm 0.31$ & $6.19 \pm 0.21$ & $7.73 \pm 0.25^{\mathrm{b}}$ & $6.47 \pm 0.26^{\mathrm{d}}$ \\
\hline
\end{tabular}

The data are expressed as the means \pm standard error (n=8/group). ${ }^{\mathrm{a}} \mathrm{P}<0.05,{ }^{\mathrm{b}} \mathrm{P}<0.01$ vs $\mathrm{Con} ;{ }^{\mathrm{c}} \mathrm{P}<0.05,{ }^{\mathrm{d}} \mathrm{P}<0.01$ vs HF. Epididymal fat was considered to be the weight of epididymal fat (g/100 g body weight). Con, control diet; $\mathrm{CP}$, control diet with polydatin supplementation; HF, high fat diet; HP, high fat diet with polydatin supplementation; ALT, alanine aminotransferase; AST, aspartate aminotransferase; TG, triglyceride; TC, total cholesterol; FFA, free fatty acids.
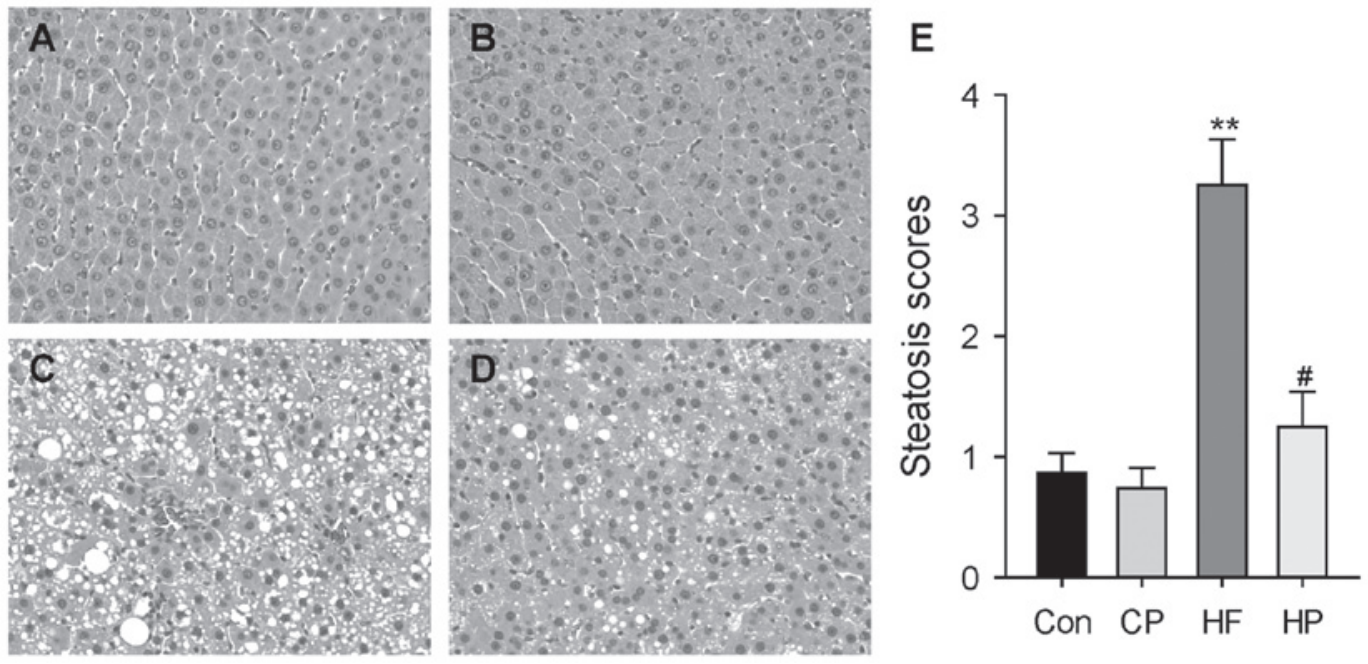

Figure 1. Effects of polydatin supplementation on high-fat diet-induced fatty liver. (A-D) Liver histologies of the representative rats from each experimental group, as observed by hematoxylin \& eosin staining of liver sections. Original magnification, x40. (A) Control diet (Con); (B) control diet supplemented with polydatin (CP); (C) high-fat diet (HF); (D) high-fat diet supplemented with polydatin (HP). (E) Liver histology scores. Steatosis was expressed as a score between 0 and 4 . The data are presented as the means \pm standard error. ${ }^{* *} \mathrm{P}<0.01$ vs Con; ${ }^{*} \mathrm{P}<0.01$ vs HF.

as compared with the control group. However, polydatin supplementation markedly improved the higher HOMA-IR, impaired glucose tolerance and increased FFA levels in the rats, following a high-fat diet $(\mathrm{P}<0.05)$.

Polydatin improves adipokine production. To determine the effects of polydatin on adipokine production, the circulating levels of insulin, leptin and adiponectin were measured. High-fat diet feeding resulted in a significant increase in plasma insulin and leptin levels $(\mathrm{P}<0.01)$ (Fig. 3A and $\mathrm{B}$ ), as compared with the Con group; whereas the adiponectin levels were significantly decreased $(\mathrm{P}<0.01)$ (Fig. 3C). Polydatin supplementation in the drinking water resulted in the substantial attenuation of the increased insulin and leptin levels, as well as reversed the abnormal adiponectin levels $(\mathrm{P}<0.05)$.

Polydatin improves IRS-2 expression levels in the liver. The effects of polydatin supplementation were examined on insulin-signaling pathways in the liver by qPCR and western blotting. There were no marked differences in the IR and IRS-1 mRNA expression levels in the rat livers, following high-fat diet feeding (Fig. 4A and B). However, high-fat diet feeding led to a significant decrease in the hepatic mRNA and protein expression levels of IRS-2 $(\mathrm{P}<0.05)$ (Fig. 4C and $\mathrm{D})$. In response to polydatin supplementation, the 
A

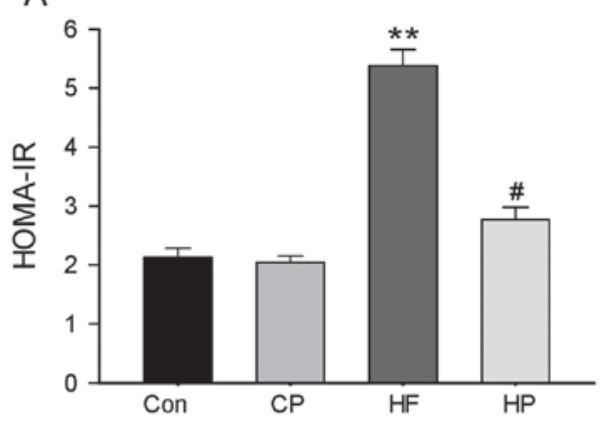

B

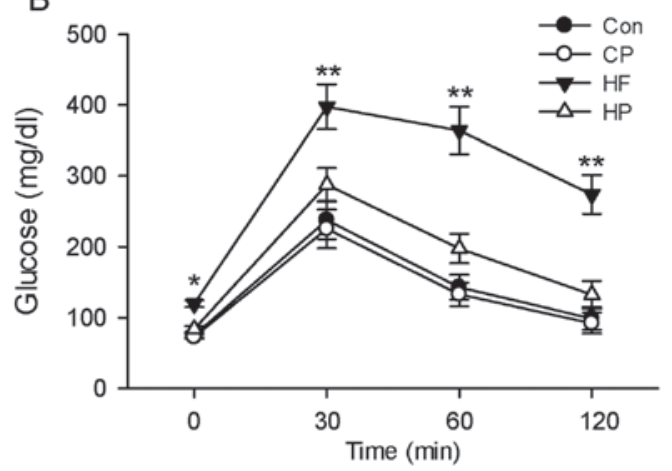

Figure 2. Effects of polydatin supplementation on insulin resistance in rats fed a high-fat diet. (A) Homeostasis model assessment of basal insulin resistance (HOMA-IR). (B) Glucose tolerance test. The data are presented as the means \pm standard error ( $\mathrm{n}=8 /$ group). ${ }^{*} \mathrm{P}<0.05$ and ${ }^{* *} \mathrm{P}<0.01$ vs con, ${ }^{*} \mathrm{P}<0.01 \mathrm{vs} \mathrm{HF}$. Con, control diet; CP, control diet supplemented with polydatin; HF, high-fat diet; HP, high-fat diet supplemented with polydatin; min, minutes.

A

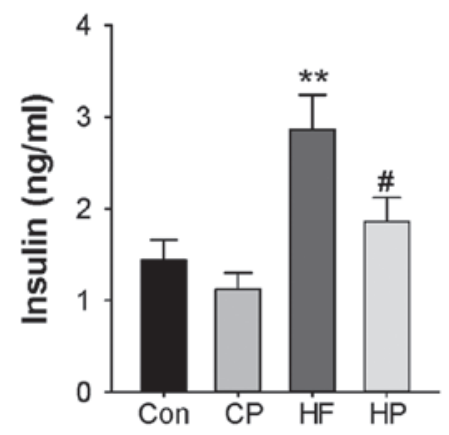

B

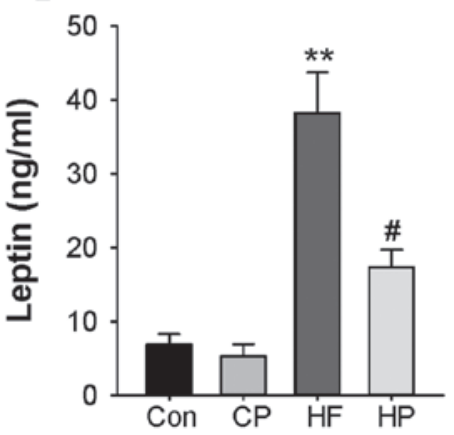

C

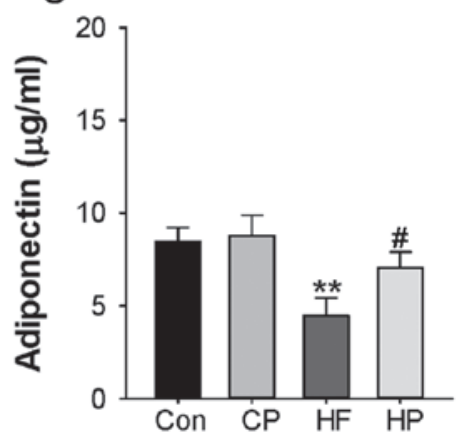

Figure 3. Effects of polydatin supplementation on adipokine production in rats fed a high-fat diet. (A) Circulating insulin levels. (B) Circulating leptin levels. (C) Circulating adiponectin levels. The rats were fed a high-fat diet with/without polydatin supplementation (1\%) in the drinking water for 12 wk. The data are presented as the means \pm standard error ( $\mathrm{n}=8 /$ group). ${ }^{* *} \mathrm{P}<0.01$ vs Con, ${ }^{\sharp} \mathrm{P}<0.01$ vs HF. Con, control diet; $\mathrm{CP}$, control diet supplemented with polydatin; HF, high-fat diet; HP, high-fat diet supplemented with polydatin.

suppressive effects of the high-fat diet on both mRNA and protein expression levels of IRS-2 were attenuated. The effects of polydatin were also determined on Akt phosphorylation status in the liver of rats fed a high-fat diet. Polydatin supplementation significantly improved the Akt phosphorylation in the rat liver, following a high-fat $\operatorname{diet}(\mathrm{P}<0.05)($ Fig. 4E).

\section{Discussion}

The present study demonstrated that polydatin supplementation alleviated insulin resistance and advanced hepatic steatosis induced by a high-fat diet in rats. These beneficial effects were shown to be associated with increased mRNA and protein expression levels of IRS-2 in the liver, and improved abnormal adipokine production. To the best of our knowledge, this is the first study to report the effects of polydatin on insulin resistance and hepatic steatosis.

Polydatin is one of the main compounds present in Polygonum cuspidatum, a plant with both medicinal and nutritional value. Zhang et al (13) previously reported that polydatin possesses in vivo protective effects against CCl4-induced liver injury in mice. Polydatin has also been shown to reduce body weight and improve dyslipidemia in high-fat diet-fed hamsters and rabbits $(15,16)$. The results of the present study demonstrated that polydatin has similarly beneficial effects on rats with NAFLD induced by a high-fat diet. Polydatin significantly decreased the plasma levels of TC, TG and FFA, and alleviated fatty liver. In accordance with the alleviations observed in the histological analyses of polydatin-treated rats, liver function was also markedly improved by polydatin supplementation. Polydatin was also shown to significantly decrease the weight of the epididymal fat in the rats. These results support the notion that polydatin effectively reduces visceral fat weight and hepatic fat content. The mechanisms by which polydatin decreases serum and hepatic lipids is yet to be fully elucidated. Usually, a reduction in fat accumulation is accomplished through decreasing food consumption. However, food consumption did not significantly differ among the high-fat diet-fed groups, in the present study. These results suggest that food and energy intake did not contribute to the significant beneficial effects of dietary polydatin. It may be hypothesized that the hypolipidemic effects of polydatin are caused by altered hepatic lipid metabolism. Previous reports have shown that polydatin may improve hyperlipidemia due to its effects on the sterol regulatory element binding protein transcription factors, which are important in the regulation of enzymes associated with lipid metabolism in vivo (17). Consistent with this report, the present study showed that polydatin decreased the weight of 
A

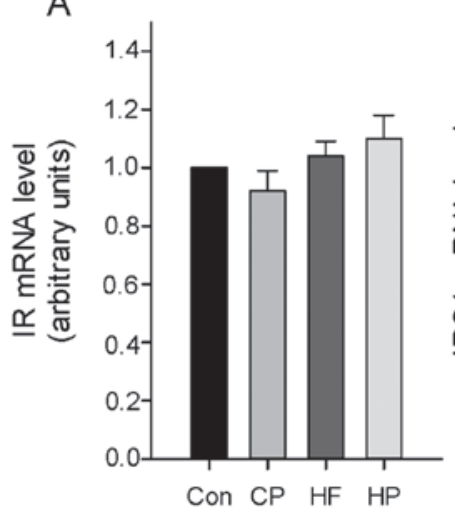

B

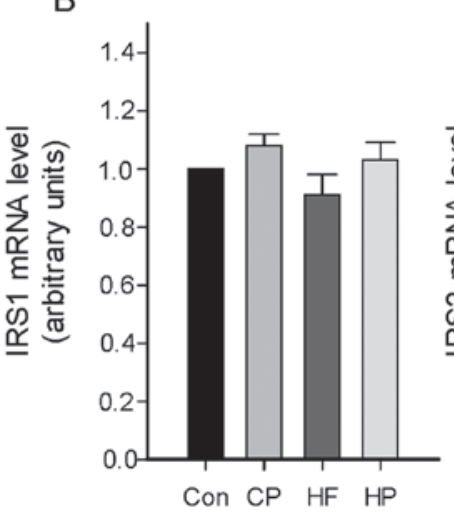

C

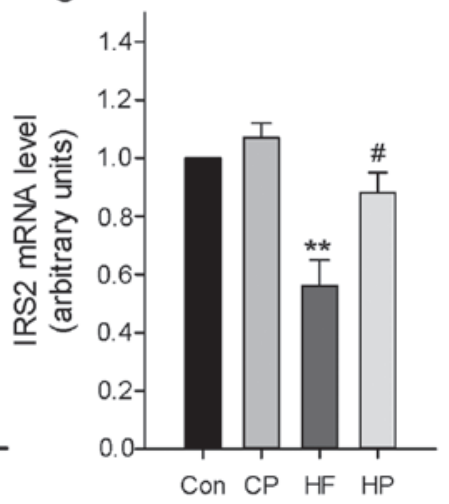

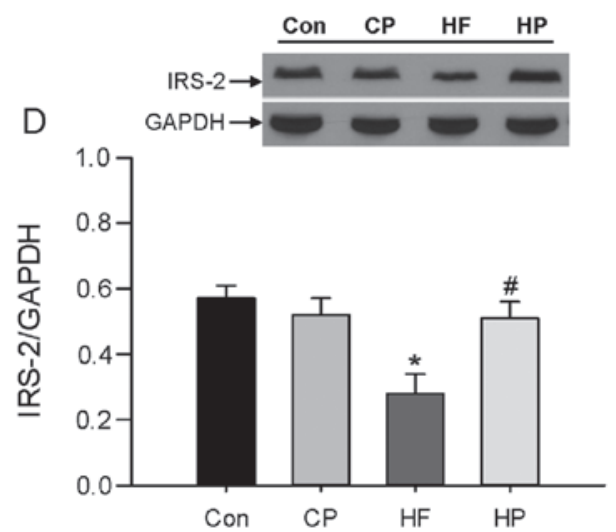

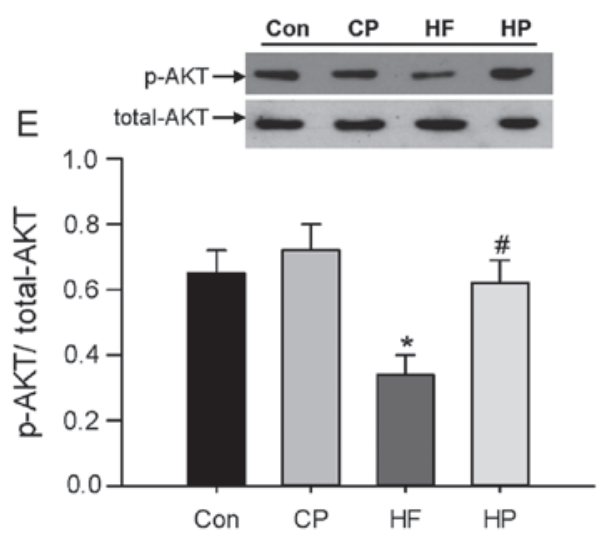

Figure 4. Effects of polydatin supplementation on hepatic insulin signaling in rats fed a high-fat diet. (A) Insulin receptor (IR), (B) Insulin receptor substrate (IRS)-1 and (C) -2 mRNA expression levels in the liver were examined by quantitative polymerase chain reaction. (D) IRS-2 protein expression levels in liver were examined by western blotting. (E) Akt phosphorylation levels in the liver were examined by western blotting. The data are presented as the means \pm standard error ( $\mathrm{n}=8$ /group). ${ }^{*} \mathrm{P}<0.05$ and ${ }^{* *} \mathrm{P}<0.01$ vs Con, ${ }^{\sharp} \mathrm{P}<0.01$ vs HF. Con, control diet; CP, control diet supplemented with polydatin; HF, high-fat diet; HP, high-fat diet supplemented with polydatin.

fat tissue and reduced the accumulation of lipids in the liver induced by NAFLD.

NAFLD is unique among the liver diseases since its etiology is closely associated with metabolic syndromes. The majority of the increased prevalence of NAFLD is due to obesity and insulin resistance (5). It has previously been reported that in the absence of obesity, even in patients with total lipodistrophy, insulin resistance leads to hepatic steatosis $(8,23)$. The mechanisms underlying the association of insulin resistance with hepatic steatosis remain unclear; however, altered insulin sensitivity has been shown to increase hepatic de novo lipogenesis and induce lipolysis of adipocyte TGs and the flux of FFA to the liver $(5,23)$. In the present study, it was demonstrated that long-term high-fat diet feeding resulted in a significant increase in hepatic fat accumulation and plasma ALT and AST levels, which are key indicators of liver injury. These changes were associated with an increased gain in body weight and systemic insulin resistance, which manifested as a significantly elevated HOMA-IR and circulating FFA levels, as well as impaired blood glucose clearance, as compared with the rats fed the control diet. Polydatin supplementation was shown to alleviate hepatic steatosis and lower plasma ALT and AST levels, as compared with the high-fat group, which was accompanied by improved HOMA-IR, circulating FFA levels and glucose tolerance test, without affecting body weight changes and daily food consumptions. These results suggest that polydatin may act as an insulin sensitizer.
In the liver, insulin has a crucial role in mediating carbohydrate and lipid homeostasis by stimulating glycogen synthesis, lipogenesis, lipoprotein synthesis, and suppressing gluconeogenesis, and very low density lipoprotein secretion in the fed state (24). The binding of insulin to the extracellular domain of its receptor, which is present on the plasma membrane of target cells, activates its intrinsic cytoplasmic tyrosine kinase activity (25). The activated insulin receptor phosphorylates tyrosine of various substrates, including IRS 1-3 and Shc $(24,25)$. Tyrosine-phosphorylated IRS proteins function as signaling molecules that propagate insulin action through binding of Src homology 2 domain-containing proteins. These include the p85 regulatory subunit of PI3K, Nck, Fyn, Grb-2, and SHP2, which mediate various aspects of insulin action $(26,27)$. PI3K is a well-characterized downstream effector of the IRS proteins (28). PI3K associates with tyrosine-phosphorylated IRS proteins following insulin stimulation, and catalyzes the formation of phosphatidylinositol-3,4,5-trisphosphate, which in turn stimulates phosphoinositide-dependent kinase activity. This results in the initiation of the activation of the downstream effector Akt. Activation of Akt leads to glucose transport and protein and glycogen synthesis (29).

Hepatic insulin resistance is an important pathophysiological feature of type II diabetes mellitus and the metabolic syndrome (30). Decreased IRS-2 expression levels, and the resultant impairment of PI3K/Akt signalling, has previously been observed in the livers of animal models for insulin 
resistance, including ob/ob and lipodystrophic mice $(31,32)$. Knockdown of IRS-2 gene expression in mice resulted in the presentation of a phenotype resembling type II diabetes in humans $(33,34)$; however, this phenotype was not induced in mice lacking IRS1 expression $(35,36)$. It has also been reported that insulin-induced PI3K and glycogen synthase activities were markedly reduced in IRS-2 null hepatocytes, and reconstitution with IRS-2 led to the recovery of the response to insulin (37). These studies indicate that insulin resistance may be mediated through inhibition of IRS-2. In the present study, the effects of polydatin supplementation on insulin sensitivity in the liver were determined by detecting the expression of IRS and Akt kinase, which are major downstream kinases activated by insulin binding to its receptors. High-fat diet feeding decreased IRS-2 and phosphorylated-Akt protein expression levels in the liver, which is indicative of dampened insulin signal transduction in the liver. Notably, the present study showed that the suppression of both kinases was rescued by polydatin supplementation in the drinking water, suggesting that polydatin may act directly on the liver tissue to prevent insulin resistance induced by a high-fat diet.

As well as insulin resistance, another critical mechanism implicated in the pathogenesis of NAFLD is the dysregulated production of adipokines. Adipokines are associated with the pathogenesis of NAFLD, through their metabolic and pro-/anti-inflammatory activities $(38,39)$. Previous evidence showed that leptin is capable of promoting insulin resistance and hepatocyte injury/fibrogenesis in both cell cultures and animal models (40). In animal models, decreased adiponectin levels are associated with hepatic steatosis and inflammation in NAFLD $(39,41)$. In the present study, the circulating levels of leptin and adiponectin were measured in rats that were fed a high-fat diet, with or without polydatin supplementation. The long-term high-fat diet feeding significantly increased the circulating levels of leptin, whereas adiponectin levels were significantly decreased, as compared with the control rats. Furthermore, the changes to adipokine production following high-fat feeding were improved by polydatin supplementation, implying that polydatin may not only prevent early stage steatosis, but also the transition to nonalcoholic steatohepatitis and fibrosis.

In conclusion, the present study was the first, to the best of our knowledge, to demonstrate that long-term feeding of a high-fat diet to rats induces fatty liver and liver injury, which were associated with obesity, insulin resistance, and dysregulated adipokine production. Polydatin supplementation in the drinking water resulted in the alleviation of hepatic steatosis and liver injury, which were associated with improved insulin resistance and abnormal adipokine production. The results of the present study suggest that polydatin may be an effective hepatoprotective agent, and a potential candidate for the treatment of fatty liver disease and insulin resistance.

\section{Acknowledgements}

The present study was supported by the National Natural Science Foundation of China (nos. 31171101 and 81100175) and the Key Project of Chinese Ministry of Education (no. 212173).

\section{References}

1. Angulo P: Nonalcoholic fatty liver disease. N Engl J Med 346: 1221-1231, 2002

2. Clark JM, Brancati FL and Diehl AM: Nonalcoholic fatty liver disease. Gastroenterology 122: 1649-1657, 2002.

3. Falck-Ytter Y, Younossi ZM, Marchesini G and McCullough AJ: Clinical features and natural history of nonalcoholic steatosis syndromes. Semin Liver Dis 21: 17-26, 2001.

4. Guilherme A, Virbasius JV, Puri V and Czech MP: Adipocyte dysfunctions linking obesity to insulin resistance and type 2 diabetes. Nat Rev Mol Cell Biol 9: 367-377, 2008.

5. Gariani K, Philippe J and Jornayvaz FR: Non-alcoholic fatty liver disease and insulin resistance: from bench to bedside. Diabetes Metab 39: 16-26, 2013.

6. Marchesini G, Bugianesi E, Forlani G, et al: Nonalcoholic fatty liver, steatohepatitis, and the metabolic syndrome. Hepatology 37: 917-923, 2003.

7. Jou J, Choi SS and Diehl AM: Mechanisms of disease progre ssion in nonalcoholic fatty liver disease. Semin Liver Dis 28 : 370-379, 2008

8. Tilg H and Moschen AR: Insulin resistance, inflammation, and non-alcoholic fatty liver disease. Trends Endocrinol Metab 19: 371-379, 2008.

9. Kimura Y: Pharmacological studies on resveratrol. Methods Find Exp Clin Pharmacol 25: 297-310, 2003.

10. Xue L: Progress in the pharmacological study of Chinese herbal drug: Polygonum cuspidatum. Zhongguo Zhong Yao Za Zhi 25: 651-653, 2000 (In Chinese).

11. Luper S: A review of plants used in the treatment of liver disease: part two. Altern Med Rev 4: 178-188, 1999.

12. Zhang H, Dou C and Gu F: Advances in the study on pharmacological actions of Polygonum cuspidatum Sieb. et Zucc.: clearing heat and detoxication. Zhong Yao Cai 26: 606-610, 2003 (In Chinese)

13. Zhang $\mathrm{H}, \mathrm{Yu} \mathrm{CH}$, Jiang YP, et al: Protective effects of polydatin from Polygonum cuspidatum against carbon tetrachloride-induced liver injury in mice. PLoS One 7: e46574, 2012.

14. Ji H, Zhang X, Du Y, Liu H, Li S and Li L: Polydatin modulates inflammation by decreasing NF- $\kappa \mathrm{B}$ activation and oxidative stress by increasing Gli1, Ptch1, SOD1 expression and ameliorates blood-brain barrier permeability for its neuroprotective effect in pMCAO rat brain. Brain Res Bull 87: 50-59, 2012.

15. Du J, Sun LN, Xing WW, et al: Lipid-lowering effects of polydatin from Polygonum cuspidatum in hyperlipidemic hamsters. Phytomedicine 16: 652-658, 2009.

16. Xing WW, Wu JZ, Jia M, Du J, Zhang H and Qin LP: Effects of polydatin from Polygonum cuspidatum on lipid profile in hyperlipidemic rabbits. Biomed Pharmacother 63: 457-462, 2009.

17. Zhang J, Tan Y, Yao F and Zhang Q: Polydatin alleviates non-alcoholic fatty liver disease in rats by inhibiting the expression of TNF- $\alpha$ and SREBP-1c. Mol Med Rep 6: 815-820, 2012.

18. Brunt EM, Janney CG, Di Bisceglie AM: Neuschwander-Tetri BA and Bacon BR: Nonalcoholic steatohepatitis: a proposal for grading and staging the histological lesions. Am J Gastroenterol 94: 2467-2474, 1999.

19. Folch J, Lees M and Sloane Starley GH: A simple method for the isolation and purification of total lipides from animal tissues. J Biol Chem 226: 497-509, 1957.

20. Zhang Q, Zhao Y, Zhang DB and Sun LJ: Effect of Sinai san decoction on the development of non-alcoholic steatohepatitis in rats. World J Gastroenterol 11: 1392-1395, 2005.

21. Matthews DR, Hosker JP, Rudenski AS, Naylor BA, Treacher DF and Turner RC: Homeostasis model assessment: insulin resistance and beta-cell function from fasting plasma glucose and insulin concentrations in man. Diabetologia 28: 412-419, 1985.

22. Zhang Q, Yao F, Raizada MK, O'Rourke ST and Sun C: Apelin gene transfer into the rostral ventrolateral medulla induces chronic blood pressure elevation in normotensive rats. Circ Res 104: 1421-1428, 2009.

23. Bugianesi E, McCullough AJ and Marchesini G: Insulin resistance: a metabolic pathway to chronic liver disease. Hepatology 42: 987-1000, 2005.

24. Saltiel AR and Kahn CR: Insulin signalling and the regulation of glucose and lipid metabolism. Nature 414: 799-806, 2001.

25. Youngren JF: Regulation of insulin receptor function. Cell Mol Life Sci 64: 873-891, 2007. 
26. Khan AH and Pessin JE: Insulin regulation of glucose uptake: a complex interplay of intracellular signalling pathways. Diabetologia 45: 1475-1483, 2002.

27. Le Roith D and Zick Y: Recent advances in our understanding of insulin action and insulin resistance. Diabetes Care 24: 588-597, 2001.

28. Cantley LC: The phosphoinositide 3-kinase pathway. Science 296: 1655-1657, 2002.

29. Wang Q, Somwar R, Bilan PJ, et al: Protein kinase B/Akt participates in GLUT4 translocation by insulin in L6 myoblasts. Mol Cell Biol 19: 4008-4018, 1999.

30. Boura-Halfon S and Zick Y: Phosphorylation of IRS proteins, insulin action, and insulin resistance. Am J Physiol Endocrinol Metab 296: E581-E591, 2009.

31. Kerouz NJ, Hörsch D, Pons S and Kahn CR: Differential regulation of insulin receptor substrates-1 and -2 (IRS-1 and IRS-2) and phosphatidylinositol 3-kinase isoforms in liver and muscle of the obese diabetic (ob/ob) mouse. J Clin Invest 100: 3164-3172, 1997.

32. Shimomura I, Matsuda M, Hammer RE, et al: Decreased IRS-2 and increased SREBP-1c lead to mixed insulin resistance and sensitivity in livers of lipodystrophic and ob/ob mice. Mol Cell 6: 77-86, 2000.

33. Previs SF, Withers DJ, Ren JM, White MF and Shulman GI Contrasting effects of IRS-1 versus IRS-2 gene disruption on carbohydrate and lipid metabolism in vivo. J Biol Chem 275: 38990-38994, 2000
34. Withers DJ, Gutierrez JS, Towery H, et al: Disruption of IRS-2 causes type 2 diabetes in mice. Nature 391: 900-904, 1998.

35. Rother KI, Imai Y, Caruso M, Beguinot F, Formisano P and Accili D: Evidence that IRS-2 phosphorylation is required for insulin action in hepatocytes. J Biol Chem 273: 17491-17497, 1998.

36. Kido Y, Burks DJ, Withers D, et al: Tissue-specific insulin resistance in mice with mutations in the insulin receptor, IRS-1, and IRS-2. J Clin Invest 105: 199-205, 2000.

37. Valverde AM, Burks DJ, Fabregat I, et al: Molecular mechanisms of insulin resistance in IRS-2-deficient hepatocytes. Diabetes 52: 2239-2248, 2003

38. Farrell GC and Larter CZ: Nonalcoholic fatty liver disease: from steatosis to cirrhosis. Hepatology 43: S99-S112, 2006.

39. Musso G, Gambino R, Durazzo M, et al: Adipokines in NASH postprandial lipid metabolism as a link between adiponectin and liver disease. Hepatology 42: 1175-1183, 2005.

40. Gambino R, Cassader M, Pagano G, Durazzo M and Musso G: Polymorphism in microsomal triglyceride transfer protein: a link between liver disease and atherogenic postprandial lipid profile in NASH? Hepatology 45: 1097-1107, 2007.

41. Wang Z, Yao T, Pini M, Zhou Z, Fantuzzi G and Song Z: Betaine improved adipose tissue function in mice fed a high-fat diet: a mechanism for hepatoprotective effect of betaine in nonalcoholic fatty liver disease. Am J Physiol Gastrointest Liver Physiol 298: G634-G642, 2010 